\title{
ON AKCOGLU AND SUCHESTON'S OPERATOR CONVERGENCE THEOREM IN LEBESGUE SPACE
}

\author{
RYOTARO SATO
}

\begin{abstract}
Let $T$ be a bounded linear operator on an $L_{1}$-space and $\tau$ its linear modulus. It is proved that if the adjoint of $\tau$ has a strictly positive subinvariant function then the following two conditions are equivalent: (i) $T^{n}$ converges weakly; (ii) $(1 / n) \sum_{i=1}^{n} T^{k_{i}}$ converges strongly for any strictly increasing sequence $k_{1}, k_{2}, \cdots$ of nonnegative integers.
\end{abstract}

1. Introduction. Let $(X, \mathscr{M}, m)$ be a $\sigma$-finite measure space and $L_{p}(X)=L_{p}(X, \mathscr{M}, m), 1 \leqq p \leqq \infty$, the usual (complex) Banach spaces. If $A \in \mathscr{M}$ then $1_{A}$ is the indicator function of $A$ and $L_{p}(A)$ denotes the Banach space of all $L_{p}(X)$-functions that vanish a.e. on $X-A$. Let $T$ be a bounded linear operator on $L_{1}(X)$ and $\tau$ its linear modulus [2]. Thus $\tau$ is a positive linear operator on $L_{1}(X)$ such that

$$
\|\tau\|_{1}=\|T\|_{1} \quad \text { and } \quad \tau g=\sup \left\{|T f| ; f \in L_{1}(X) \text { and }|f| \leqq g\right\}
$$

for any $0 \leqq g \in L_{1}(X)$. The adjoint of $T$ is denoted by $T^{*}$. Clearly $T$ is a contraction if and only if $\tau^{*} 1 \leqq 1$. In [1] Akcoglu and Sucheston proved that if $T$ is a contraction then the following two conditions are equivalent: (i) $T^{n}$ converges weakly; (ii) $(1 / n) \sum_{i=1}^{n} T^{k_{i}}$ converges strongly for any strictly increasing sequence $k_{1}, k_{2}, \cdots$ of nonnegative integers. In this note we shall prove that if $\tau^{*}$ has a strictly positive subinvariant function in $L_{\infty}(X)$ then the equivalence of (i) and (ii) still holds. Applying this result, we obtain that if $T$ is a positive linear operator on $L_{1}(X)$ such that $\sup _{n}\left\|(1 / n) \sum_{k=0}^{n-1} T^{k}\right\|_{1}<\infty$ and also such that $T^{n} f$ converges weakly for any $f \in L_{1}(X)$ with $\int f d m=0$ and if $T^{*}$ has a strictly positive subinvariant function in $L_{\infty}(X)$, then for any $f \in L_{1}(X)$ with $\int f d m=0$ and any strictly increasing sequence $k_{1}, k_{2}, \cdots$ of nonnegative integers, $(1 / n) \sum_{i=1}^{n} T^{k_{i}}$ converges strongly. This is a generalization of another result of Akcoglu and Sucheston [1].

Received by the editors January 17, 1973.

AMS (MOS) subject classifications (1970). Primary 47A35.

Key words and phrases. Bounded linear operator, linear modulus of a bounded linear operator, weak and strong convergence.

(c) American Mathematica 1 Society 1973 
2. Results. Throughout this section we shall assume that there exists a strictly positive function $s \in L_{\infty}(X)$ with $\tau^{*} s \leqq s$. In the proofs we shall also assume that $(X, \mathscr{M}, m)$ is a finite measure space, since the $L_{1}$ of a $\sigma$-finite measurc space is isometric to the $L_{1}$ of a finite measure space (cf. [1]).

THEOREM 1. The following two conditions are equivalent:

(i) If $f \in L_{1}(X)$ then $T^{n} f$ converges weakly;

(ii) If $f \in L_{1}(X)$ then $(1 / n) \sum_{i=1}^{n} T^{k_{i}}$ converges strongly for any strictly increasing sequence $k_{1}, k_{2}, \cdots$ of nonnegative integers.

Proof. We first prove that (i) implies (ii). For $s f \in L_{1}(X)$, where $f \in$ $L_{1}(X)$, define $V(s f)=s T f$. Since $\left\{s f ; f \in L_{1}(X)\right\}$ is a dense subspace of $L_{1}(X)$ in the norm topology and $\|V(s f)\|_{1} \leqq\|s f\|_{1}$ (cf. [3]), $V$ may be considered to be a linear contraction on $L_{1}(X)$. Since $V^{n}(s f)=s T^{n} f$ for any $n \geqq 0$ and $T^{n} f$ converges weakly, it follows that $V^{n}(s f)$ converges weakly. Thus, since $V$ is a contraction, it is easily seen that for any $A \in \mathscr{M}$ the limit $\mu(A)=\lim _{n} \int_{A} V^{n} f d m$ exists. Since the measure $m$ is finite, the VitaliHahn-Saks theorem implies that $\mu$ is a countably additive measure on $\mathscr{M}$ absolutely continuous with respect to $m$. Therefore there exists a function $g \in L_{1}(X)$ such that $\mu(A)=\int_{A} g d m$ for any $A \in \mathscr{M}$. It follows that $V^{n} f$ converges weakly to $g$. Thus, by Theorem 2.1 of [1], for any $f \in L_{1}(X)$ and any strictly increasing sequence $k_{1}, k_{2}, \cdots$ of nonnegative integers,

$$
\frac{1}{n} \sum_{i=1}^{n} V^{k_{i}}(s f)=\frac{1}{n} s\left(\sum_{i=1}^{n} T^{k_{i}} f\right)
$$

converges strongly. Let $\lim _{n}\left\|(1 / n) s\left(\sum_{i=1}^{n} T^{k i} f\right)-f_{0}\right\|_{1}=0$ for some $f_{0} \in$ $L_{1}(X)$ and let $\varepsilon>0$ be arbitrarily fixed. Since $T^{n} f$ converges weakly, there exists a positive number $\delta$ such that $A \in \mathscr{M}$ and $m(A)<\delta$ imply $\int_{A}\left|T^{n} f\right| d m<\varepsilon$ for any $n \geqq 0$. Choose $\eta>0$ such that $m(\{x ; s(x)<\eta\})<\delta$ and $\int_{\{x ; s(x)<\eta\}}\left|f_{0}\right| d m<\varepsilon$, and put $A=\{x ; s(x)<\eta\}$. Then

$$
\begin{aligned}
\left\|\frac{1}{n} \sum_{i=1}^{n} T^{k_{i}} f-\frac{1}{m} \sum_{j=1}^{m} T^{k_{j}} f\right\|_{1} & \left\|\frac{1}{n} \sum_{i=1}^{n} 1_{A} T^{k_{i}} f\right\|_{1}+\left\|\frac{1}{m} \sum_{j=1}^{m} 1_{A} T^{k_{j}} f\right\|_{1} \\
& +\left\|\frac{1}{n} \sum_{i=1}^{n} 1_{X-A} T^{k_{i}} f-\frac{1}{m} \sum_{j=1}^{m} 1_{X-A} T^{k_{j}} f\right\|_{1} \\
< & 2 \varepsilon+\left\|\frac{1}{n} \sum_{i=1}^{n} 1_{X-A} T^{k_{i}} f-1_{X-A} \frac{1}{s} f_{0}\right\|_{1} \\
& +\left\|\frac{1}{m} \sum_{j=1}^{m} 1_{X-A} T^{k_{j}} f-1_{X-A} \frac{1}{s} f_{0}\right\|_{1}
\end{aligned}
$$


and

$$
\begin{aligned}
\left\|\frac{1}{n} \sum_{i=1}^{n} 1_{X-A} T^{k_{i}} f-1_{X-A} \frac{1}{s} f_{0}\right\|_{1} \\
\quad \leq \frac{1}{\eta}\left\|\frac{1}{n} s\left(\sum_{i=1}^{n} 1_{X-A} T^{k_{i}} f\right)-1_{X-A} f_{0}\right\|_{1} \rightarrow 0
\end{aligned}
$$

as $n \rightarrow \infty$, from which we observe that $(1 / n) \sum_{i=1}^{n} T^{k_{i}} f$ is a Cauchy sequence in $L_{1}(X)$, and hence $(1 / n) \sum_{i=1}^{n} T^{k_{i}} f$ converges strongly.

Conversely if (ii) holds, then it follows easily that $\sup _{n}\left\|T^{n}\right\|_{1}<\infty$ and that for any $f \in L_{1}(X)$ and any $A \in \mathscr{M}, \lim _{n} \int_{A} T^{n} f d m$ exists, and hence $T^{n} f$ converges weakly. This completes the proof of Theorem 1 .

THEOREM 2. Let $T$ be a positive linear operator on $L_{1}(X)$ with

$$
\sup _{n}\left\|\frac{1}{n} \sum_{k=0}^{n-1} T^{k}\right\|_{1}<\infty
$$

and suppose $T^{*} s \leqq s$ for some $0<s \in L_{\infty}(X)$. Then the following two conditions are equivalent:

(i) If $f \in L_{1}(X)$ and $\int f d m=0$, then $T^{n} f$ converges weakly;

(ii) If $f \in L_{1}(X)$ and $\int f d m=0$, then for any strictly increasing sequence $k_{1}, k_{2}, \cdots$ of nonnegative integers, $(1 / n) \sum_{i=1}^{n} T^{k_{i}} f$ converges strongly.

Proof. Suppose (i) holds. It is known [3] that if $T$ has no nontrivial nonnegative invariant function in $L_{1}(X)$, then the operator $V$ introduced above also has no nontrivial nonnegative function in $L_{1}(X)$. Thus it follows from [1] that, if $T^{n} f$ converges weakly then

$$
\lim _{n}\left\|V^{n}(s f)\right\|_{1}=\lim _{n}\left\|s T^{n} f\right\|_{1}=0 .
$$

Let $\varepsilon>0$ be arbitrarily fixed, and let $\delta$ be a positive number such that $A \in \mathscr{M}$ and $m(A)<\delta$ imply $\int_{A}\left|T^{n} f\right| d m<\varepsilon$ for any $n \geqq 0$. Choose $\eta>0$ such that $m(\{x ; s(x)<\eta\})<\delta$, and put $A=\{x ; s(x)<\eta\}$. Then

$$
\begin{aligned}
\left\|T^{n} f\right\|_{1} & \leqq\left\|1_{A} T^{n} f\right\|_{1}+\eta^{-1}\left\|1_{X-A} s T^{n} f\right\|_{1} \\
& <\varepsilon+\eta^{-1}\left\|s T^{n} f\right\|_{1}
\end{aligned}
$$

and $\left\|s T^{n} f\right\| \rightarrow 0$ as $n \rightarrow \infty$, thus $\lim _{n}\left\|T^{n} f\right\|_{1}=0$.

If there exists $0 \leqq h \in L_{1}(X)$ with $\|h\|_{1}>0$ and $T h=h$, then it follows from [1] that for any $f \in L_{1}, T^{n} f$ converges weakly. Thus the strong convergence of $(1 / n) \sum_{i=1}^{n} T^{k_{i}} f$ for any strictly increasing sequence $k_{1}, k_{2}, \cdots$ of nonnegative integers follows from Theorem 1 .

Clearly (ii) implies (i), and the proof is complete. 


\section{BIBLIOGRAPHY}

1. M. Akcoglu and L. Sucheston, On operator convergence in Hilbert space and in Lebesgue space, Periodica Math. Hungarica 2 (1972), 235-244.

2. R. V. Chacon and U. Krengel, Linear modulus of a linear operator, Proc. Amer. Math. Soc. 15 (1964), 553-559. MR 29 \#1543.

3. R. Sato, Ergodic properties of bounded $L_{1}-$ operators, Proc. Amer. Math. Soc. 39 (1973), 540-546.

Department of Mathematics, Josai University, Sakado, Saitama 350-02, Japan 